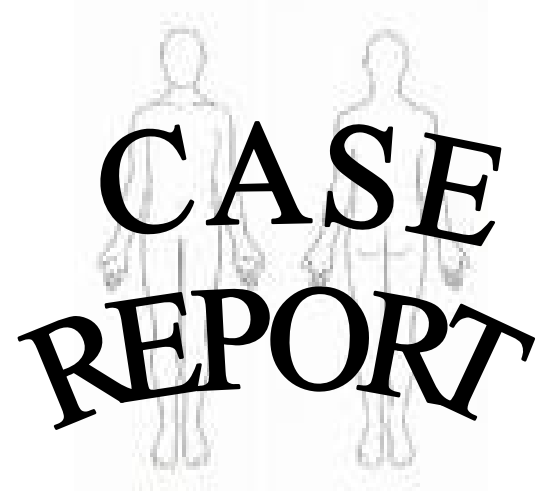

\section{Utilization of Fosphenytoin for Digoxin-Induced} Ventricular Arrhythmia

Jessica R. Newman, D.O. ${ }^{1}$

Joshua R. Howitt, Pharm.D. ${ }^{2}$

Trenton D. Nauser, M.D. ${ }^{1,2}$

${ }^{1}$ University of Kansas Medical Center

Department of Internal Medicine, Kansas City, KS

${ }^{2}$ Kansas City Veterans Administration Medical Center

Kansas City, MO

\section{Introduction}

Digoxin is a class-IV anti-arrhythmic which has indications for use in congestive heart failure and atrial dysrhythmias including atrial fibrillation, atrial flutter, and atrial tachycardia. ${ }^{1}$ It was prescribed first by Dr. William Withering for hydrops pectoris and described in An Account of the Foxglove and Some of Its Medical Uses in $1785 .{ }^{2}$ Though digoxin has been used for over 200 years, its properties and benefits continue to be investigated. Digoxin inhibits the sodium-potassium ATPase pump, thereby increasing intracellular sodium-calcium exchange in the cardiac myocyte. The resultant increase in intracellular calcium causes increased contractility. Digoxin also exerts an anti-adrenergic action in patients with heart failure by inhibiting sympathetic outflow. ${ }^{1,3}$

While benefits of digoxin therapy were anecdotal for decades, in the early 1990s, PROVED, RADIANCE, and DIG trials showed prevention of clinical deterioration, decrease in hospitalizations, and improved exercise tolerance in digoxin treated patients despite the absence of an absolute survival advantage. ${ }^{4-6}$ Notwithstanding these proven benefits, digoxin utility has been restricted by a narrow therapeutic window. Most cases of toxicity involve serum digoxin levels greater than two ng/mL, although digoxin's interaction with many commonly used medications, including but not limited to verapamil, diltiazem, erythromycin, and tetracycline may precipitate toxicity at therapeutic serum drug levels. The mechanism of action may involve precipitating the AV-blocking effect (e.g., verapamil, diltiazem) or increasing the bioavailability of digoxin (e.g., macrolide antibiotics). Advanced age, renal failure, ischemic heart disease, cardiomyopathy, and electrolyte disturbances including hypokalemia, hypomagnesemia, and hypercalcemia also may predispose to toxicity. Toxicity may lead to neurologic, gastrointestinal, and/or cardiac symptoms, including headaches, dizziness, ataxia, yellow-green chromatopsia, nausea, vomiting, diarrhea, various cardiac dysrhythmias, and cardiac death.,

Though gastrointestinal manifestations are often the first sign of digoxin toxicity, patients may present with cardiac arrhythmias which rapidly can progress to a fatal arrhythmia if unrecognized. Premature ventricular beats or atrioventricular block are the earliest and most common abnormal rhythms found in up to $30-40 \%$ of verified cases of toxicity. ${ }^{7}$ Up to $80-90 \%$ of toxicity cases involve some type of cardiac arrhythmia. ${ }^{8}$ Treatment can prove challenging and may need to be multifaceted due to the many potential manifestations of digoxin toxicity. Supportive care, correction of electrolyte disturbances and use of digoxin-specific antibody Fab fragments to bind free digoxin 
and aid in excretion are well documented. Fab fragments are pieces of the antibody that contain the antigen binding site.

Treatment of dysrhythmias, if persistent despite administration of digoxin-specific antibody Fab fragments, is less standardized. ${ }^{8}$ Research has investigated the use of phenytoin to slow the development of digoxin-induced arrhythmias. The suspected mechanism of action involves a suppression of central sympathetic outflow, thereby decreasing ventricular automaticity. ${ }^{8}$ The utility of fosphenytoin, the pro-drug of phenytoin, for treatment of digoxin-induced arrhythmias has not been established.

\section{Case Report}

A 78-year-old Caucasian male was admitted with digoxin toxicity. The patient was unable to provide a history. His wife had observed one week of progressive weakness, lethargy, confusion, and anorexia. His past medical history was significant for ischemic cardiomyopathy with an ejection fraction of $10 \%$, for which he took digoxin $0.125 \mathrm{mg}$ daily. He also had mitral valve regurgitation, hyperlipidemia, hypertension, hypothyroidism, and peptic ulcer disease. In addition to digoxin, his home medications included carvedilol, clopidogrel, furosemide, spironolactone, potassium chloride, gemfibrozil, hydrochlorothiazide/lisinopril, levothyroxine, and omeprazole.

The patient was afebrile, with a pulse of 59 , blood pressure of $105 / 45 \mathrm{mmHg}$, respiratory rate of 8 , and oxygen saturation of $99 \%$ on two liters per minute of oxygen via nasal canula. The physical examination revealed a notably thin individual. He was alert, however, oriented only to person and place. His heart rate was bradycardic with a regular rhythm; no murmur, rub or gallop was present. Serum chemistry revealed: BUN $193 \mathrm{mg} / \mathrm{dL}$, creatinine $11.9 \mathrm{mg} / \mathrm{dL}$, potassium $8 \mathrm{mEq} / \mathrm{L}$, calcium $9.4 \mathrm{mg} / \mathrm{dL}$, and magnesium $2.9 \mathrm{mg} / \mathrm{dL}$. The digoxin level was elevated at $4.5 \mathrm{ng} / \mathrm{mL}$.

The initial electrocardiogram showed a left bundle branch block, unchanged from the month prior. Chest radiograph showed cardiomegaly, without pulmonary vascular congestion.

Intravenous fluids, albuterol solution via nebulizer, intravenous insulin with 50\% dextrose and oral sodium polystyrene sulfonate (SPS) were administered to treat the hyperkalemia. Since the patient's hyperkalemia responded to medical treatment, emergent dialysis was not performed. Confusion and hyperkalemia were presumed to be consequences of digoxin toxicity and digoxin Immune Fab was administered immediately using the following dosing formula: serum digoxin concentration in $\mathrm{ng} / \mathrm{mL}$ multiplied by weight in $\mathrm{kg}$ divided by 100 .

Despite digoxin-specific antibody Fab fragments, ventricular arrhythmias commenced with premature ventricular contractions (PVC), followed by ventricular bigeminy and brief runs of wide complex bradycardia (see Figure 1). Shortly thereafter, the patient had a six beat run of ventricular tachycardia.

Intravenous phenytoin was not readily available. There was concern for use of lidocaine in the setting of progressive renal failure. Fosphenytoin was given in a bolus at a dose of $20 \mathrm{mg} / \mathrm{kg} \mathrm{IV}$. Within one hour of the loading dose, a significant decrease in PVCs, bigeminy, and ectopy was noted (see Figure 2). Digoxin Immune Fab treatment was repeated. Fosphenytoin was continued every 12 hours for the remainder of the hospitalization. Telemetry remained stable with infrequent PVCs over the duration of the hospital stay.

Despite medical management of electrolytes and fluid balance, the patient's renal function and uremia worsened and hemodialysis was initiated late on hospital day two. Despite hemodialysis, the patient 


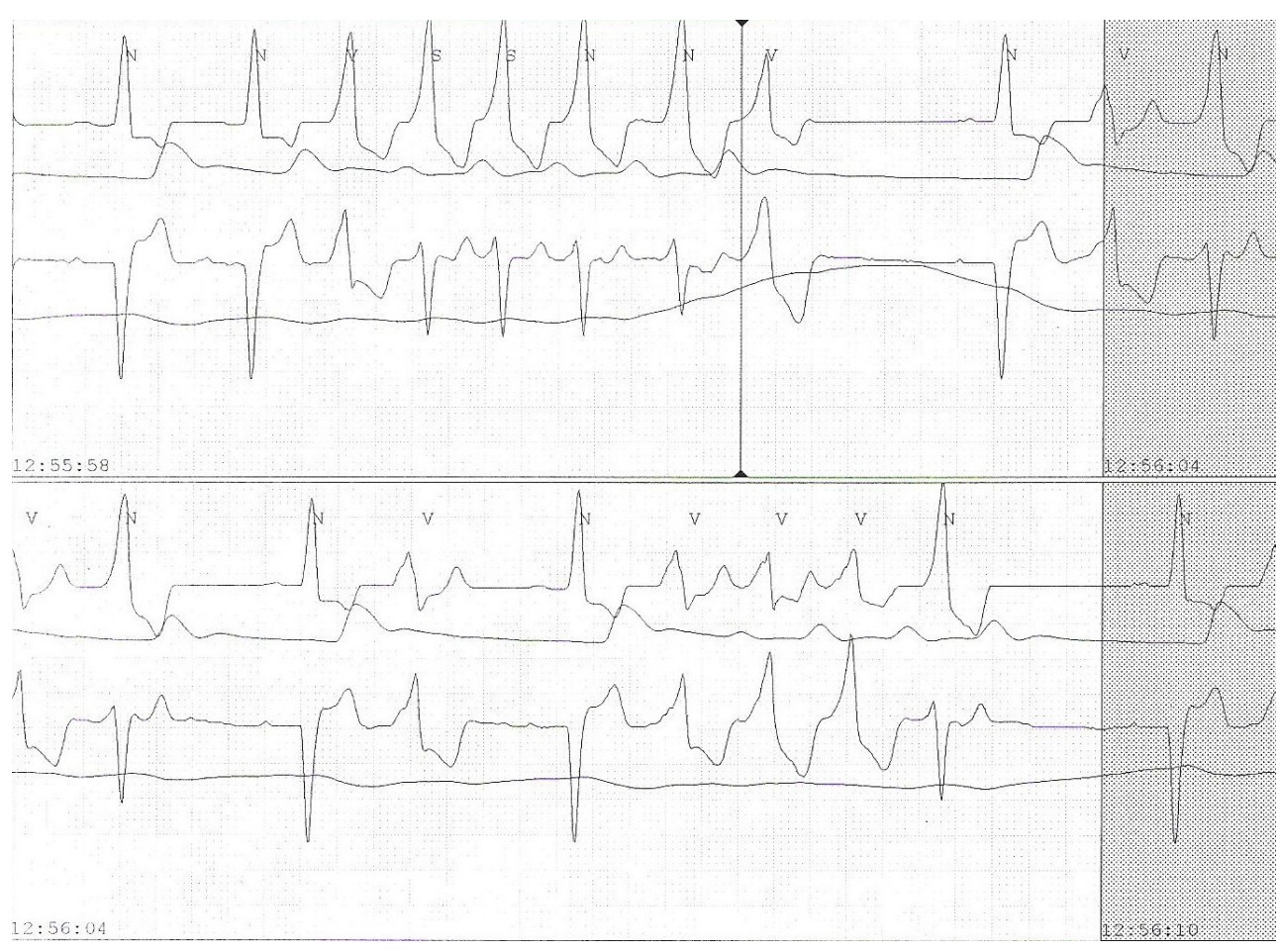

Figure 1. Telemetry demonstrating non-sustained ventricular tachycardia in addition to multiple premature ventricular beats.

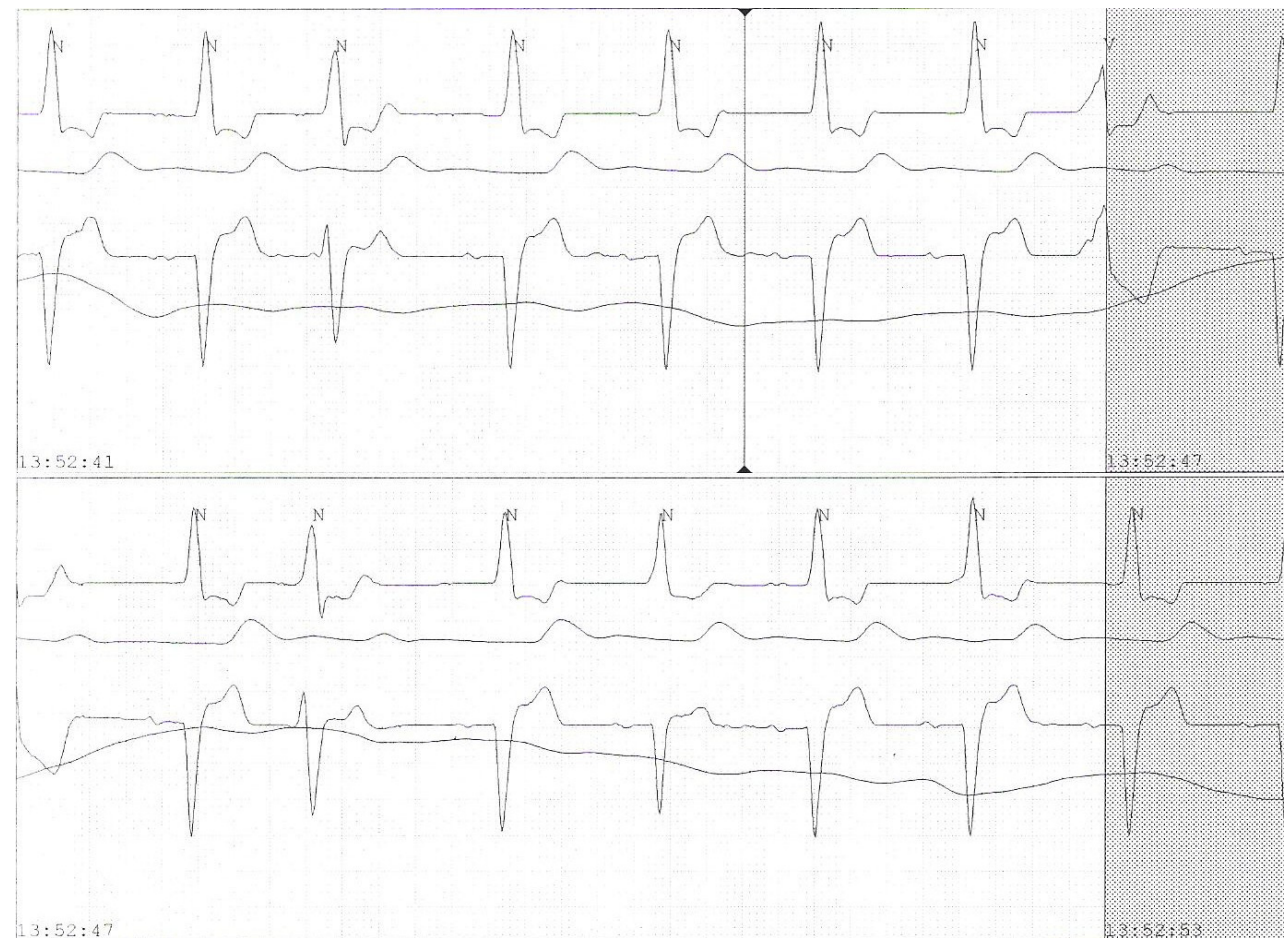

Figure 2. Telemetry demonstrating a substantial decrease in ventricular ectopy less than one hour after fosphenytoin loading. 
remained somnolent and confused. A decision was made by the family to change goals of care to comfort measures, and the patient expired on hospital day three.

\section{Discussion}

Historically, digoxin has been implicated as one of the most common causes of adverse drug reaction. ${ }^{9}$ Toxicity can be acute or chronic and symptoms of digoxin toxicity include gastrointestinal upset, neurologic findings, including visual disturbance and confusion, and cardiac dysrhythmia. $^{10}$ In $10-15 \%$ of cases of documented toxicity, an ectopic cardiac rhythm is the first sign of intoxication. ${ }^{7}$

Digoxin-induced arrhythmia occurs due to a depression of the sinoatrial node and its conduction which can lead to atrioventricular (AV) block and ventricular ectopy. In turn, this can lead to increased automaticity, extra-systoles and tachyarrhythmia induced by the initiation of ectopic atrial pacemakers. While PVCs, AV block, atrial tachycardia, and ventricular ectopy commonly are identified rhythms in digoxin toxicity, there are more specifically associated arrhythmias, including bidirectional ventricular tachycardia usually resulting from an alteration of the intraventricular conduction pathway. Furthermore, ventricular ectopy may be more common in those patients with preexisting heart disease, as in our patient. ${ }^{7}$

In this case, ventricular arrhythmias started with PVCs, followed by ventricular bigeminy and brief runs of wide complex tachycardia. The presence of a non-sustained ventricular tachycardia prompted initiation of antiarrhythmic treatment. In digoxininduced arrhythmia, Class IA agents such as procainamide are contraindicated due to their impact of decreasing conduction, thereby propagating AV block. In cases of severe bradyarrhythmia, atropine can be useful. The first line agents to treat ventricular ectopy are phenytoin and lidocaine; phenytoin has been shown to be more effective. ${ }^{7,11}$

Phenytoin's efficacy in suppressing cardiac ectopy is proposed to be related to its effect on resting membrane potential, the action potential amplitude, and the upstroke velocity in phase 0 of the cardiac cycle. In the presence of a low serum potassium, phenytoin can increase the action potential of both atrial and Purkinje fibers, enhancing conduction and increasing the phase 0 upstroke velocity. Less is known about the effect of phenytoin on a reentrant circuit in the presence of normal serum potassium. (In our patient, serum potassium had been normalized by the time of fosphenytoin administration).

Phenytoin may improve conduction of premature impulses and in the setting of digoxin toxicity depress spontaneous diastolic depolarization. Though phenytoin has been effective for ventricular ectopy associated with digoxin overdose, little effect has been seen in treating atrial arrhythmia or ventricular arrhythmia in the setting of chronic cardiac disease. ${ }^{11}$ The reported dose of phenytoin is $250 \mathrm{mg}$ IV over 10 minutes which can be repeated in boluses of $100 \mathrm{mg}$ every five minutes up to one gram. ${ }^{7}$ Intravenous phenytoin must be used with caution in patients with preexisting hypotension and may cause hypotension if it is administered at rates exceeding $50 \mathrm{mg} / \mathrm{min}$. Fosphenytoin for the treatment of digoxin-induced cardiac arrhythmia has not been reported. ${ }^{7}$

Fosphenytoin is a pro-drug of phenytoin, hydrolyzed into phenytoin in-vivo. Benefits of intravenous fosphenytoin treatment as compared to intravenous phenytoin are related to an increased water-solubility, thus decreasing injection site reactions and allowing faster administration. The intravenous preparation of phenytoin contains approximately $40 \%$ propylene glycol in 
addition to ethanol, leading to an alkaline $\mathrm{pH}$ of 12 . Fosphenytoin, with a $\mathrm{pH}$ of 8.8 , is compatible with most intravenous fluids. ${ }^{12}$ The propylene glycol in intravenous phenytoin has been shown in some cases to lead to increased hypotension and cardiac arrhythmia in studies on acute seizure

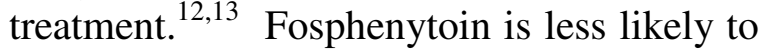
cause hemodynamic instability. The lack of immediate availability of intravenous phenytoin and the ability to infuse fosphenytoin more rapidly prompted treatment with this agent. This resulted in a substantial reduction in the patient's ventricular ectopy, presumably by the same antiarrhythmic mechanism as phenytoin. He was treated with fosphenytoin through the duration of his hospitalization with no known direct complications of therapy.

\section{Conclusions}

This case was a 78-year-old male with digoxin toxicity who developed ventricular arrhythmias. The presence of the elevation of free digoxin with the risk of further dysrhythmia precludes the use of many traditional antiarrhythmics. This patient's deteriorating renal function cautioned use of lidocaine. At this institution, limited availability of intravenous phenytoin prompted use of intravenous fosphenytoin which decreased the ventricular ectopy. Administration of intravenous fosphenytoin, shown to result in fewer incidences of infusion site phlebitis and rate-dependent hypotension as compared to intravenous phenytoin, may be an alternative therapy in an attempt to suppress ventricular ectopy associated with digoxin toxicity.

\section{References}

${ }^{1}$ Gheorghiade M, Adams Jr KF, Colucci WS. Digoxin in the management of cardiovascular disorders. Circulation 2004; 109:2959-2964.
${ }^{2}$ Withering W. An account of the foxglove and some of its medical uses, with practical remarks on dropsy and other diseases. In: FA Willius, TE Keys (Eds). Classics of Cardiology: A Collection Of Classic Works on the Heart and Circulation with Comprehensive Biographic Accounts of the Authors. Malabar, FL: Krieger, 1983.

${ }^{3}$ Haji SA, Movahed A. Update on digoxin therapy in congestive heart failure. Am Fam Physician 2000; 62:409-418.

${ }^{4}$ Uretsky BF, Young JB, Shahidi FE, Yellen LG, Harrison MC, Jolly MK. Randomized study assessing the effect of digoxin withdrawal in patients with mild to moderate chronic congestive heart failure: Results of the PROVED trial. PROVED Investigative Group. J Am Coll Cardiol 1993; 22:955-962.

${ }^{5}$ Packer M, Gheorghiade M, Young JB, et al. Withdrawal of digoxin from patients with chronic heart failure treated with angiotensin-converting-enzyme inhibitors. RADIANCE Study. N Engl J Med 1993; 329:1-7.

6 The effect of digoxin on mortality and morbidity in patients with heart failure. The Digitalis Investigation Group. N Engl J Med 1997; 336:525-533.

${ }^{7}$ Litonjua MR, Penton S, Robinson C, Daubert GP. Digoxin: The monarch of cardiac toxicities. J Pharm Pract 2005; 18:157-168.

${ }^{8}$ Bhatia SJ. Digitalis toxicity - Turning over a new leaf? West J Med 1986; 145:74-82.

${ }^{9}$ Williamson KM, Thrasher KA, Fulton $\mathrm{KB}$, et al. Digoxin toxicity: An evaluation in current clinical practice. Arch Intern Med 1998; 158:2444-2449.

${ }^{10}$ Smith TW, Antman EM, Friedman PL, Blatt CM, Marsh JD. Digitalis glycosides: Mechanisms and manifestations of toxicity. Part III. Prog Cardiovasc Dis 1984; 27:21-56. 
${ }^{11}$ Wit AL, Rosen MR, Hoffman BF. Electrophysiology and pharmacology of cardiac arrhythmias. VIII. Cardiac effects of diphenylhydantoin. B. Am Heart J 1975; 90:397-404.

${ }^{12}$ Fisher JH, Patel TV, Fisher PA. Fosphenytoin: Clinical pharmacokinetics and comparative advantages in the acute treatment of seizures. Clin Pharmacokinet 2003; 42:33-58.
${ }^{13}$ DeToledo JC, Ramsay RE. Fosphenytoin and phenytoin in patients with status epilepticus: Improved tolerability versus increased costs. Drug Saf 2000; 22:459466.

Keywords: fosphenytoin, digoxin, drug toxicity, cardiac arrhythmia, case report 